\title{
Improving the ECG classification of inferior and lateral myocardial infarction by inversion of lead aVR
}

\author{
I B A Menown, A A J Adgey
}

\begin{abstract}
Objective-To assess whether the use of inverted lead aVR ( $-a V R)$ would improve the classification of acute inferior or lateral myocardial infarction presenting with ST elevation.

Design-Observational study. The presence of $\geqslant 1 \mathrm{~mm}$ ST elevation in lead $-a V R$ (derived by manual assessment of ST depression in conventional lead aVR) was determined by a single investigator, blinded to patient outcome.

Patients -173 consecutive patients with chest pain for $\leqslant 12$ hours and ST elevation of $\geqslant 1 \mathrm{~mm}$ in inferior leads (II, III, aVF) or lateral leads (I, aVL, V5, V6), excluding those with anterolateral ST elevation.

Main outcome measure-Incidence of ST elevation in lead -aVR in patients with inferior or lateral ST elevation, or both.

Results-ST elevation in lead $-a V R$ was present in 25 of 136 patients (18\%) with inferior but no lateral ST elevation (indicating greater superior involvement) and in three of 11 patients $(27 \%)$ with lateral but no inferior ST elevation (indicating greater inferior involvement). ST elevation in lead $-a V R$ bridged the gap between inferior and lateral ST elevation in 15 of 25 $(60 \%)$ patients with inferior and lateral chest lead (V5/V6) ST elevation, and in all patients with inferior and lateral limb lead (I/aVL) ST elevation. The presence of ST elevation in lead $-a V R$ was associated with a larger infarct size as defined by median peak creatine kinase on serial sampling: $1780 v 987 \mathrm{mmol} / \mathrm{l} ; \mathrm{p}=0.021$.

Conclusions-Use of lead - $a V R$ improves the ECG classification of acute inferior or lateral acute myocardial infarction and thus may be useful as part of the routine 12 lead ECG assessment of such patients.

(Heart 2000;83:657-660)
\end{abstract}

Keywords: electrocardiography; acute myocardial infarction

The conventional ECG samples body surface potentials from the horizontal axis in an orderly fashion from right to left across the body surface. Thus in a normal subject, an orderly progression in ECG waveform morphologyfor example, $\mathrm{R}$ wave amplitude-is seen from lead V1 to V6. In contrast, the conventional ECG samples the frontal axis in a less orderly fashion (fig $1 \mathrm{~A}$ ) with a $60^{\circ}$ gap between lead I and lead II, and a $90^{\circ}$ gap between lead III and lead aVR. Furthermore, the conventional ECG displays first the bipolar leads (I, II, III) and then the unipolar leads (aVR, aVL, aVF), and thus no clear progression in waveform morphology is seen.

However, display of lead aVR $\left(-150^{\circ}\right)$ in inverted format as lead $-a V R\left(+30^{\circ}\right)$ bridges the gap between lead I $\left(0^{\circ}\right)$ and lead II $\left(60^{\circ}\right)$. Use of $-a V R$ thus enables the frontal axis to be viewed in a logical or orderly display of $30^{\circ}$ intervals from aVL (left superior) to lead III (right inferior). ${ }^{1}$ In patients with acute inferolateral myocardial infarction an orderly display illustrates the importance of $-a V R$ as part of the sequential rise and fall in ST segment height as the leads circle the apex of the left ventricle (fig $1 \mathrm{~B}$ ).

Frontal lead aVR $\left(-150^{\circ}\right)$ is often neglected in ECG interpretation, with clinicians effectively using an "11 lead" ECG. ${ }^{2}$ We hypothesised that display of lead aVR in inverted for- mat $\left(-a V R+30^{\circ}\right)$, enabling it to be viewed as part of a sequential display of the frontal axis, would improve the classification of acute myocardial infarction presenting with ST elevation when it affected the frontal axis, that is, inferior or lateral myocardial infarction.

\section{Methods}

SUBJECTS

Patients were recruited consecutively over a 30 month period as they presented to a 24 hour physician-manned mobile coronary care unit (cardiac ambulance), the emergency department, or in a medical ward. Inclusion criteria were as follows:

- onset of acute chest pain within the previous 12 hours;

- $\mathrm{ST}$ elevation $\geqslant 1 \mathrm{~mm}$ on the presenting 12 lead ECG in one or more inferior leads (II, III, aVF) or lateral leads (V5, V6, I, aVL);

- the diagnosis of acute myocardial infarction confirmed by a rise in creatine kinase of $>2 \times$ the upper normal laboratory reference level (with CK-MB isoenzyme activity $\geqslant 7 \%$ if the aetiology of the total creatine kinase rise was equivocal), or a rise in creatine kinase of $<2 \times$ the upper laboratory normal reference level accompanied by serial ECG changes consistent with new myocardial infarction (new $Q$ waves of $\geqslant 0.03$ seconds duration, or 
A
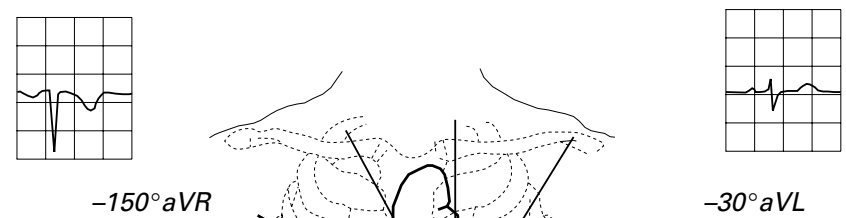

$-150^{\circ} a V R$

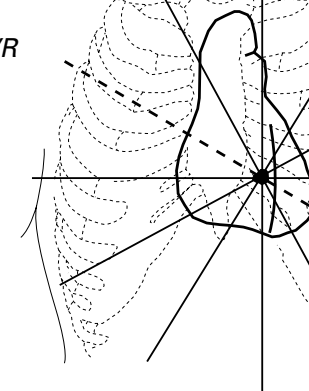

$+120^{\circ}$ lead III

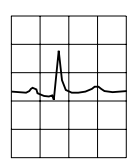

$+90^{\circ}$ aVF

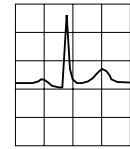

$+60^{\circ}$ lead II

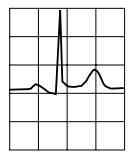

$-30^{\circ} a V L$

$0^{\circ}$ lead I

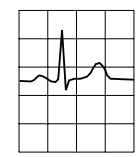

$+30^{\circ}$ "-aVR"

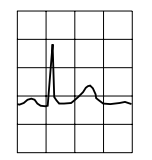

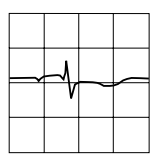

aVL

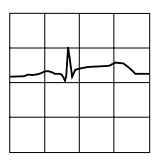

I

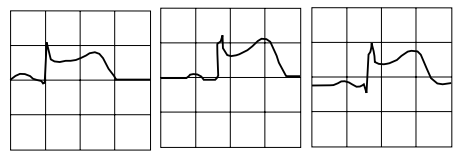

$-a V R$

II

aVF

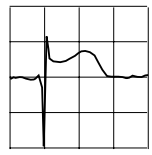

III

Figure 1 (A) Frontal axis illustrating individual leads including conventional and inverted aVR. (B) An orderly ECG display of the frontal axis in a patient with acute inferolateral myocardial infarction incorporating -aVR, arranged in $30^{\circ}$ intervals from aVL to lead III. A sequential rise and fall in ST segment height is seen as the leads circle the apex of the left ventricle.

new persistent $\mathrm{T}$ wave inversion in two or more contiguous leads, or both).

Patients with anterolateral ST elevation $(\geqslant 1 \mathrm{~mm}$ ST elevation in lateral leads and one or more of leads V1-V4) were excluded. All patients had samples obtained for creatine kinase analysis at the time of admission, the

Table 1 Baseline characteristics

\begin{tabular}{llll}
\hline & $\begin{array}{l}\text { No significant } S T \\
\text { elevation }(<1 \mathrm{~mm}) \text { in } \\
\text { lead }-a V R(n=129)\end{array}$ & $\begin{array}{l}\text { ST elevation } \geqslant 1 \mathrm{~mm} \\
\text { in lead -aVR }(n=44)\end{array}$ & Overall $(n=173)$ \\
\hline Male & $74(57)$ & $31(70)$ & $105(61)$ \\
Age (mean (SD)) & $62(10.4)$ & $61(9.2)$ & $62(10.1)$ \\
Age $\geqslant 75$ years & $14(11)$ & $2(4)$ & $16(9)$ \\
Family history of IHD & $63(49)$ & $22(50)$ & $85(49)$ \\
Current smokers & $63(49)$ & $24(54)$ & $87(50)$ \\
Diabetes & $15(12)$ & $0(0)$ & $15(9)$ \\
Hypertension & $31(24)$ & $9(20)$ & $40(23)$ \\
Hyperlipidaemia & $23(18)$ & $11(25)$ & $46(17)$ \\
Previous angina & $35(27)$ & $7(16)$ & $42(24)$ \\
Previous MI & $35(27)$ & 1.8 & 2.0 \\
Median pain to ECG & 2.1 & $34(77)$ & $130(75)$ \\
$\quad$ (hours) & & & \\
Use of fibrinolytic & $96(74)$ & & \\
$\quad$ treatment & & & \\
\hline
\end{tabular}

Values are $\mathrm{n}(\%)$ unless otherwise stated.

${ }^{\star} \mathrm{p}=0.018$.

IHD, ischaemic heart disease; MI, myocardial infarction. following morning, and daily thereafter for three days.

The study was approved by the Queen's University of Belfast research ethics committee. All patients gave informed consent.

LEAD - $a V R$ ASSESSMENT

Standard distal limb lead placement was employed in all patients whether in the ambulance or in the hospital. Although adopted in Sweden for over 20 years, ${ }^{1}$ electronic inversion of lead aVR at the time of recording is not widely available elsewhere, and was not possible using our 12 lead ECG machines. Thus $-a V R$ display (fig 1) was obtained by digital vertical inversion of a scanned image of conventional lead aVR (using Adobe Photoshop software) to aid ECG analysis of the frontal leads. However, to enable measurements to be made on the original ECG data, the presence of $\geqslant 1 \mathrm{~mm}$ ST elevation (J point $+80 \mathrm{~ms}$ ) in lead $-a V R$ was derived by manual assessment of ST depression in conventional lead aVR. ECGs were read by a single investigator, blinded to patient outcome, thereby minimising intraobserver bias and eliminating interobserver variability. All measurements were made using a Minnesota approved measuring loupe (6× EDSCORP pocket comparator) in order to reduce intraobserver error.

STATISTICAL METHODS

Baseline categorical variables were analysed by $\chi^{2}$ test and continuous clinical variables by analysis of variance. The relation of ST elevation in lead $-a V R$ to peak creatine kinase was assessed by the Mann-Whitney U test. In addition a multiple regression analysis was performed to control for the potential confounding influence of concurrent use of thrombolytic treatment or the presence of reciprocal anteroseptal ST depression. A value of $\mathrm{p}<0.05$ was taken as significant.

\section{Results}

Over the study period, 173 patients were recruited. Of these, 136 patients had inferior ST elevation only, 11 had lateral ST elevation only, and 26 had both inferior and lateral ST elevation (which involved the lateral limb leads I or aVL in four of the 26 patients and the lateral chest leads V5 or V6 in 25 of the 26). ST elevation in $-a V R$ was present in 44 of the 173 patients. Baseline characteristics are listed in table 1. Patients with and without ST elevation in $-a V R$ were comparable, with no significant differences between sets with respect to baseline demographics, risk factors for ischaemic heart disease, and cardiac history, except for an excess of diabetes in those without ST elevation in $-a V R$.

INFERIOR WITHOUT LATERAL ST ELEVATION In the 136 patients with ST elevation in the inferior leads but not in the lateral leads, 25 $(18 \%)$ had ST elevation in lead $-a V R$, indicating a greater superior involvement of the acute myocardial infarction in the frontal plane than suggested by the conventional ECG display (fig 2, arrow A). 

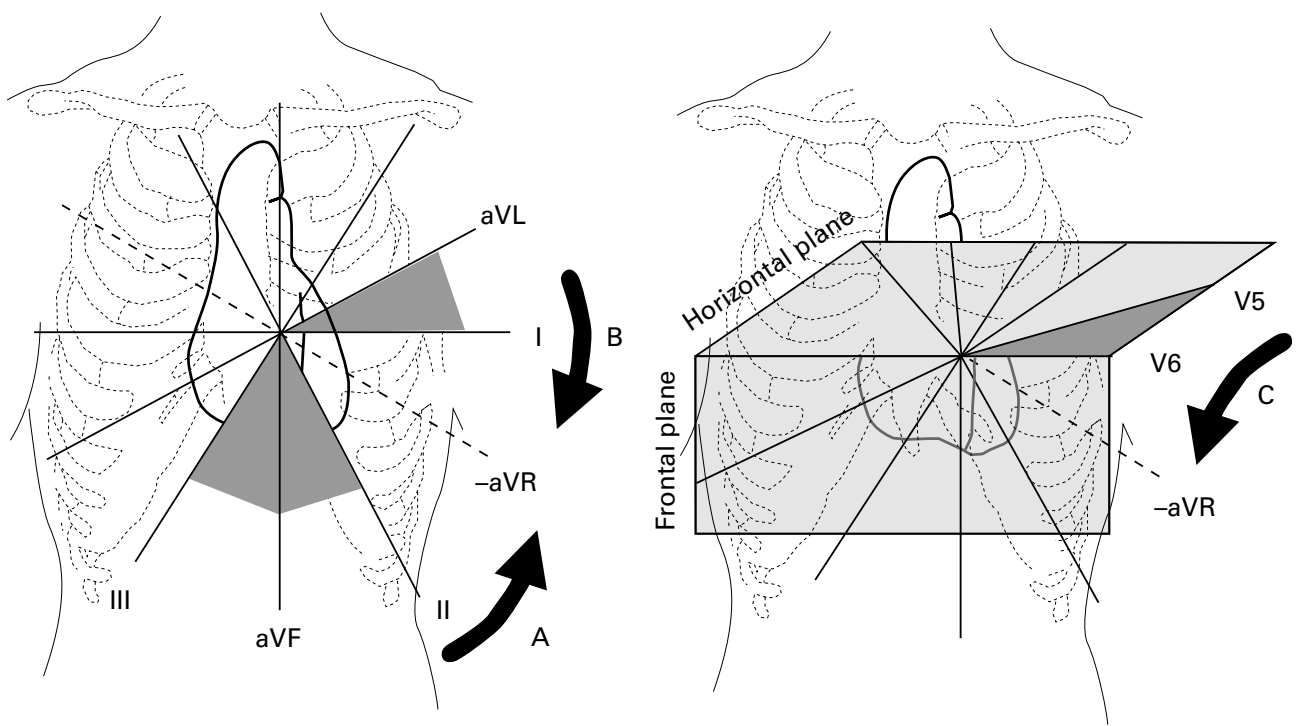

Figure 2 Diagrammatic illustration of ST elevation in lead-aVR indicating a greater superior involvement in patients with acute inferior $S T$ elevation (arrow $A$ ), and a greater inferior involvement in patients with acute lateral limb lead $S T$ elevation (arrow B) or lateral chest lead ST elevation (arrow C).

LATERAL WITHOUT INFERIOR ST ELEVATION

In the 11 patients with ST elevation in lateral leads (limb leads I, aVL, or chest leads V5 and V6) but not in inferior leads, three patients $(27 \%)$ had ST elevation in lead $-a V R$, indicating a greater inferior involvement of the acute myocardial infarction in the frontal plane than suggested by the conventional ECG display (fig 2 , arrows $\mathrm{B}$ and $\mathrm{C}$ ).

INFERIOR AND LATERAL ST ELEVATION

Of the 26 patients with ST elevation in both inferior and lateral leads, ST elevation in lead $-a V R$ bridged the gap between inferior and lateral regions in 16 patients $(62 \%)$. Of the 25 patients with ST elevation in inferior and lateral chest leads (V5 and V6), ST elevation in lead $-a V R$ bridged the gap between inferior and lateral regions in 15 patients $(60 \%)$. ST elevation in lead $-a V R$ bridged the gap between inferior and lateral regions in all four patients with inferior and lateral limb lead ST elevation (leads I and aVL).

INFARCT SIZE

The presence of ST elevation $\geqslant 1 \mathrm{~mm}$ in lead $-a V R$ was associated with a larger infarct size as defined by median peak creatine kinase on serial sampling: $1780 v 987 \mathrm{mmol} / \mathrm{l} ; \mathrm{p}=0.021$. The association of ST elevation in $-a V R$ with higher peak creatine kinase was shown by multiple regression analysis to be independent of the use of thrombolytic treatment or the presence of reciprocal anteroseptal ST depression.

\section{Discussion}

Our study has clearly shown the clinical value of assessing lead $-a V R$ as part of the evaluation of the frontal axis in patients with acute myocardial infarction presenting with inferior or lateral ST elevation. Previous studies have found lead $-a V R$ to be useful in a variety of clinical settings. Anderson and colleagues have shown it to be valuable in assessing the extent of established Q waves, ${ }^{1}$ and incorporation of lead $-a V R$ as part of an orderly display of the frontal axis can aid rapid calculation of axis angle. ${ }^{13}$ Viik and colleagues reported that ST depression in lead $-a V R$ (as part of a lead set which also included leads I, V4, V5, and V6) was useful for differentiating between patients with coronary artery disease and those with a low likelihood of disease, using exercise stress testing. ${ }^{4}$ Lead $-a V R$ may be diagnostically helpful in heart rate adjusted ST segment (ST/ HR) analysis during exercise stress testing. ${ }^{5}$ Gambill and colleagues have calculated the upper limit of normal $\mathrm{T}$ wave amplitude in lead $-a V R$ with a view to incorporating it into automated ECG analysis in patients presenting with symptoms of acute transmural ischaemia. ${ }^{6}$

DISPLAY

The failure to encourage universal adoption of the orderly ECG display of the frontal axis is most probably caused by the previous technical limitations of ECG recording systems, which have now been overcome by digital technology. ${ }^{1}$ The true display of lead $-a V R$ (fig 1 ) is helpful for a general ECG assessment as it facilitates a sequential and logical interpretation of waveform morphology. Nevertheless, detection of the converse finding in conventional lead aVR (as used in this study), is a practical alternative method, particularly if only one variable is being sought.

IMPLICATIONS FOR REPERFUSION TREATMENT Overall, patients with inferior or lateral myocardial infarction are thought to be at lower absolute risk than those with anterior myocardial infarction and derive a smaller relative risk reduction from thrombolytic treatment. ${ }^{7}$ Current practice thus tends towards use of streptokinase in patients with inferior myocardial infarction, whereas t-PA (tissue plasminogen activator) or, if available, primary angioplasty with or without stenting is increasingly being used for the "higher risk" anterior 
myocardial infarct patients. ${ }^{8}$ The use of serial creatine kinase sampling in our study was probably a suboptimal estimate of infarct size, and greater sampling frequency (for example, 4-6 hourly) to enable calculation of the area under the creatine kinase release curve may have been preferable. Nevertheless as patients with ST elevation in lead $-a V R$ in our study were shown to have a higher median peak creatine kinase even after controlling for the use of thrombolytic treatment or the presence of reciprocal anteroseptal ST depression, they may represent a higher risk subgroup who should be assigned to an "optimum" reperfusion regimen.

\section{CONCLUSIONS}

Display of lead aVR in inverted format $(-a V R)$ facilitates sequential evaluation of the frontal axis, improves the ECG classification of acute inferior or lateral acute myocardial infarction, and thus may be useful as part of the routine 12 lead ECG assessment of such patients.
IBAM was supported by a Royal Victoria Hospital clinical fellowship. We wish to thank research nurse Bernie Smith for her invaluable assistance.

1 Anderson ST, Pahlm O, Selvester RH, et al. Panoramic display of the orderly sequenced 12-lead ECG. $\mathcal{F}$ Electrocardiol 1994;27:347-52.

2 Pahlm US, Pahlm O, Wagner GS. The standard 11-lead ECG. Neglect of lead aVR in the classical limb lead display. F Electrocardiol 1996;29(suppl):270-4.

3 Dower GE, Nazzal SB, Pahlm O, et al. Limb leads of the electrocardiogram: sequencing revisited. Clin Cardiol 1990; 13:346-8.

4 Viik J, Lehtinen R, Turjanmaa V, et al. Correct utilization of exercise electrocardiographic leads in differentiation of men with coronary artery disease from patients with a low likelihood of coronary artery disease using peak exercise ST-segment depression. Am $\mathcal{F}$ Cardiol 1998;81:964-9.

5 Viik J, Lehtinen R, Turjanmaa V, et al. The effect of lead selection on traditional and heart rate-adjusted ST segment analysis in the detection of coronary artery disease during exercise testing. Am Heart f 1997;134:488-94.

6 Gambill CL, Wilkins ML, Haisty WK, et al. T wave amplitudes in normal populations. Variation with ECG lead, sex, and age. $\mathcal{F}$ Electrocardiol 1995;28:191-7.

7 Fibrinolytic Therapy Trialists' Collaborative Group. Indications for fibrinolytic therapy in suspected acute myocardial infarction: collaborative overview of early mortality and major morbidity results from all randomised trials of more than 1000 patients. Lancet 1994;343:311-22.

8 Antman EM, Braunwald E. Acute myocardial infarction In: Braunwald E, ed. Heart disease. A textbook of cardiovascular medicine. Philadelphia: WB Saunders, 1997:1184-288.

\section{Electronic pages}

\section{eHEART: www.heartjnl.com}

The following electronic only articles are published in conjunction with this issue of Heart.

\section{Multivessel coronary thrombosis, acute myocardial infarction, and no reflow in a patient with essential thrombocythaemia \\ H Terada, $H$ Satoh, $A$ Uehara}

Essential thrombocythaemia (ET) has been reported rarely to cause coronary thrombosis, but the management is still undefined. A 63 year old woman with multivessel coronary thrombosis, acute myocardial infarction (MI), and no reflow in reperfused coronary artery in association with ET is presented. The patient's platelet count was only moderately raised at the onset of MI, but peripheral blood smear and bone marrow evaluation revealed clumping giant platelets and numerous large hyperploid megakaryocytes. Long term prophylaxis with antiplatelet agents in patients with ET is recommended, even if the platelet count is not largely raised. Cytoreductive treatment may also be effective for secondary prevention when thrombotic complications occur.

(Heart 2000;83:e10) www.heartjnl.com/cgi/content/full/83/ $6 / \mathrm{e} 10$

\section{Myocardial stunning after streptokinase: what is the significance of the $Q$ wave? \\ W Keeble, W Martin, I Hutton}

A 58 year old woman presented with symptoms and electrocardiographic features consistent with acute inferoposterior myocardial infarction. The attempt at reperfusion with aspirin and thrombolytic treatment was deemed unsuccessful in view of $Q$ wave development on ECG, a 48 hour period of hypotension and oliguria, and extensive wall motion abnormality on echocardiography. This was at variance with findings of a minimal cardiac enzyme rise. On the seventh day, list mode acquired, ECG gated, cineimages of perfusion, and blood pool demonstrated strikingly normal perfusion despite continued contractile dysfunction. Six weeks later ECG, echocardiography, and radionuclide images had all normalised consistent with resolution of myocardial stunning. This practical method for the diagnosis of stunning, the significance of the ECG changes in particular the development of $\mathrm{Q}$ waves, and the time taken to resolution of this phenomenon is discussed.

(Heart 2000;83:e11) www.heartjnl.com/cgi/content/full/ $83 / 6 / \mathrm{e} 11$

Exchange of pacing or defibrillator leads following laser sheath extraction of non-functional leads in patients with ipsilateral obstructed venous access

$F$ A Bracke, L $M$ van Gelder, $N$ Sreeram, A Meijer

Occlusion of the subclavian or brachiocephalic vein in pacemaker or defibrillator patients prohibits ipsilateral implantation of new leads with standard techniques in the event of lead malfunction. Three patients are presented in whom laser sheath extraction of a non-functional lead was performed in order to recanalise the occluded vein and to secure a route for implantation of new leads. This technique avoids abandoning a useful subpectoral site for pacing or defibrillator therapy. The laser sheath does not affect normally functioning leads at the same site.

(Heart 2000;83:e12) www.heartjnl.com/cgi/content/full/ $83 / 6 / \mathrm{e} 12$ 